\title{
Cycle Selection and Neuro-Voting System for Classifying Heart Sound Recordings
}

\author{
Mostafa Abdollahpur, Shadi Ghiasi, Mohammad Javad Mollakazemi, Ali Ghaffari \\ Cardiovascular Research Group (CVRG), Department of Mechanical Engineering, \\ KN Toosi University of Technology, Tehran, Iran
}

\begin{abstract}
Aims: To address the PhysioNet/Computing in Cardiology (CinC) Challenge 2016 [1], this study aims to discuss a new method to classify PCG signals collected from a variety of clinical or nonclinical environments.

Method: The raw PCG data is pre-processed by applying a band pass finite-duration impulse response filter (FIR) and discrete wavelet transform (DWT). For PCG delineation, first (s1) and second (s2) heart sounds are detected based on springer's algorithm. In this study, three analyses are used for feature extraction. The first one is time-frequency domain analysis in which the energy of nodes of wavelet packet transform is extracted beside the features already obtained from the time domain and perceptual domain analyses. Using fisher discriminant analysis (FDA) for feature reduction, a new space to better represent features is obtained. For heart sounds classification, a combination of three classifiers of artificial neural network (ANN) are implemented. To accomplish a precise classification, the output of all classifiers are employed in a fuzzy-logic algorithm (voting system).

Results: By evaluation of the proposed method by training set the sensibility of 0.96, specificity of 0.97 and total score of 0.98 were achieved. Also, evaluation on the hidden test set resulted in the sensibility, specificity and modified accuracy of $0.811,0.872$ and 0.841 , respectively.
\end{abstract}

\section{Introduction}

Heart sound can reflect most of the heart valve disorders which can be detected by phonocardiogram signal (PCG) analysis. Sonic vibrations of heart and blood flow are recorded in PCG which can be useful for discriminating pathological heart sounds from normal ones. Extensive research has been conducted concerning the profiteering of the heart sound signals for automated detection of various heart diseases and pathological conditions. Detecting the precise location of main components of heart sounds in the presence of heart murmurs and high level measurement noises, has been a major issue. Recently, detection and boundary identification of main heart sounds in cardiac sounds using an expert frequency-energy based metric has been proposed by [2]. The introduction of probabilistic models for heart sound segmentation led to improved precision. Using these models, the springer's and Schmidt's method gave an excellent performance on a noisy dataset and can be demonstrated to be the current state-of-the-art [3-4]. The automatic detection of heart sound abnormalities strongly depends on the proper features, which mostly are related to timing, morphology, and time-frequency properties of heart sounds. In order to better detect heart murmurs a detailed analysis was carried out using spectral and energy features together (Mel Frequency Cepstral Coefficient (MFCC), Short-Time Fourier Transform (STFT), and instantaneous energy) by [5]. The classification is an important step for detection and identification of cardiac pathological conditions using cardiac sound signals. Different approaches have been done due to this end. Neural networks classifiers, SVM classifier and k-mean clustering have been commonly used.

The PhysioNet/CinC Challenge 2016 [1] presents an opportunity for researchers to develop the classification of normal and abnormal heart sound recordings.

The paper will describe first the data in section 2.1. In section 2.2.1 and 2.2.2 the preprocessing and segmentation techniques are explained. A method to choose the best cycles is proposed in section 2.2.3. A number of features and their new representation into a new space which are as an input of classifiers are presented in section 2.2.4. and 2.2.5. In section 2.2.6. a classification model and a voting system are described. Finally, results and discussions are presented in Section 4 and 5, respectively.

\section{Materials and methods}

\subsection{Data sets}

The PhysioNet/CinC Challenge 2016 provides train and test sets with a total of 3153 and 1277 PCG recordings, respectively. More about data can be found in [1]. 


\subsection{Methodology}

\subsubsection{Preprocessing}

For more accurate segmentation and feature extraction, preprocessing is performed to obtain de-noised signal using following procedure:

First, the signal is down sampled to $1 \mathrm{kHz}$. Given that the possible frequency range for cardiac sounds is between 25 and $400 \mathrm{~Hz}$, a fourth order Butterworth band pass filter with cut-off frequencies at $25 \mathrm{~Hz}$ and $400 \mathrm{~Hz}$ is applied to the raw signal. In order to remove spikes which are higher in amplitude than the heart sounds a four-stage algorithm proposed by Schmidt is used to identify and remove them [4]. Then, the normalization stage is applied to map the amplitudes to a reference line. Finally, a soft threshold denoising technique suggested by [6] is applied.

\subsubsection{Segmentation}

Prior to any other event, the exact location of the main components amongst the heart sound events which are S1 and S2 should be detected. In order to do the accurate segmentation of these sounds in noisy real-world datasets from normal and abnormal heart sound recordings, the automatic segmentation algorithm proposed by Springer is used [3]. This algorithm is based on HSMM with the use of logistic regression for emission probability estimation.

\subsubsection{Cycle selection}

For a better reliability of features, a novel method is proposed. As a result of consulting with cardiologists, in most cardiac diseases cardiac abnormalities show themselves in all heart beats in PCG signals. Based on this fact, a new method is presented to classify high quality cycles from cycles that are contaminated with noises whose frequency domain is the same as the main components of PCG. Also some cycles may be not be properly segmented. Thereby, instead of considering all cycles, only cycles that are segmented correctly with less amount of noise are qualified for feature extraction. This procedure is done based on the energy of each intra beat segment.

In Figure 1 the original signal (a) and the springer's segment (b) according to an open-source algorithm which is available in PhysioNet are shown. As it is obvious in (b) there is a mistake in detecting S2 because the end of the systole is detected as S2. Also in the last cycle part of S2 is segmented as systole. This will mostly affect the extracted features related to energy, entropy and also duration-dependent features. These cycles are ignored for next steps and the final signal composing of high quality cycles is demonstrated in (c).

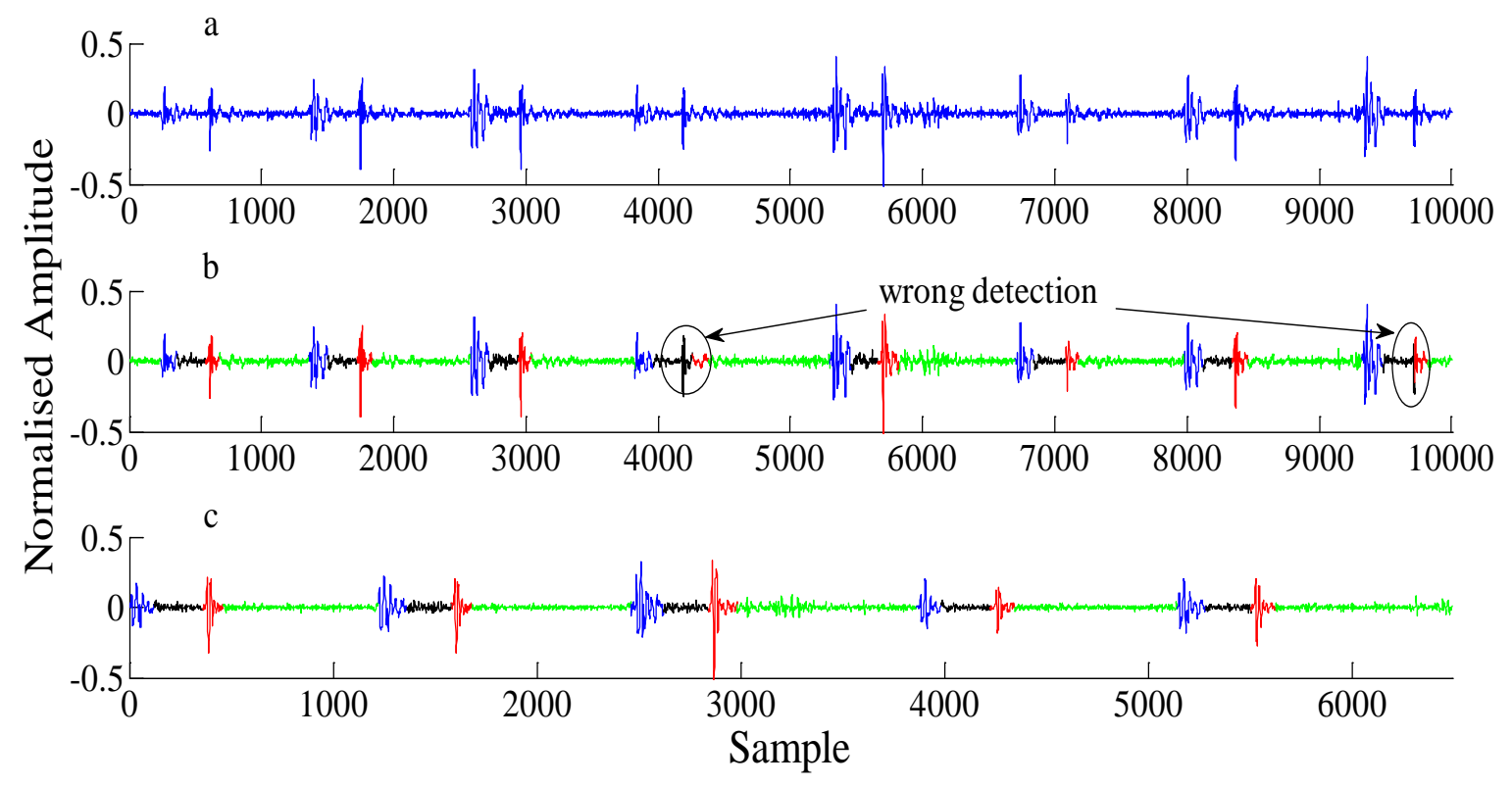

Figure 1. a) original signal after preprocessing b) springer's segmentation algorithm for detecting four main components c) applying cycle selection method to choose high quality cycles . the four main componnets of heart sound are shown in diffrenet colors as: Blue:S1, black:systole, red:S2, green:diastole 


\subsubsection{Feature extraction}

In this section a group of features which best highlight the discrimination between normal and abnormal signals are extracted. Therefore, 5 groups of features from different domains are used. Unlike other studies, to deal with the conditions of systolic and diastolic murmurs the features within the entire signal as well as separately in systolic and diastolic segments are obtained and used for classification. A summary of these features are enlisted in Table 1.

Table 1 . The list of the extracted features.

\begin{tabular}{|c|c|c|c|}
\hline $\begin{array}{l}\text { Feature } \\
\text { domain }\end{array}$ & Description & $\begin{array}{l}\text { Number } \\
\text { of } \\
\text { features }\end{array}$ & Source \\
\hline $\begin{array}{l}\text { Time } \\
\text { domain }\end{array}$ & $\begin{array}{l}9 \text { energy values } \\
\text { from the } \\
\text { envelope at } \\
\text { specific times } \\
\text { between main } \\
\text { states }\end{array}$ & 9 & [7] \\
\hline \multirow{3}{*}{$\begin{array}{l}\text { Time- } \\
\text { frequency }\end{array}$} & $\begin{array}{l}\text { Mean values } \\
\text { and ratios of } \\
\text { power and } \\
\text { Shannon } \\
\text { entropy of } \\
\text { systole and } \\
\text { diastole for each } \\
\text { selected cycle }\end{array}$ & 4 & $\begin{array}{l}\text { This } \\
\text { paper }\end{array}$ \\
\hline & $\begin{array}{l}\text { the absolute } \\
\text { sum over } 9 \\
\text { intervals from } \\
\text { the } \\
\text { decomposition } \\
\text { of the } 6^{\text {th }} \\
\text { wavelet detail of } \\
\text { a level } 10 \\
\text { daubechies } 2\end{array}$ & 9 & [8] \\
\hline & $\begin{array}{l}\text { the entropy of } \\
\text { the wavelet } \\
\text { approximation } \\
\text { of each wavelet } \\
\text { detail using } \\
\text { previous mother } \\
\text { wavelet }\end{array}$ & 11 & [8] \\
\hline $\begin{array}{l}\text { Perceptual } \\
\text { features }\end{array}$ & $\begin{array}{l}\text { Mean of the } 14 \\
\text { MFCC } \\
\text { coefficients } \\
\text { extracted for } 4 \\
\text { main } \\
\text { components }\end{array}$ & 56 & [7] \\
\hline
\end{tabular}

\subsubsection{Feature reduction}

In this study, the number of the features which are extracted for classification is 89 which were explained in the previous section. The high number of features will result in computational complexity of the classifiers and some features are mutually correlated. In some cases, these redundant features cause the adverse effects on the classifier performance. Thereby, for improving the accuracy of the classifier and reducing the computational fallacy of the algorithm, the feature vector is reduced. To meet this end, (Fisher Discriminant Analysis) FDA is employed. With the use of FDA, we are able to map features into a new space where by only one or two dimensions the discrimination between normal and abnormal signals are apparent.

\subsubsection{Classification}

The classification procedure can be summarized into 3 steps:

1. Using FDA: A matrix that represents mapping of features is obtained. This matrix is valuable since it can detect most of normal signals alone. This happens when the features obtained in the previous section map to a two dimensional space and for normal signals these new dimensions lie between a threshold which is obtained by try and error.

2. Using neural networks: In this section neural networks with supervised training algorithms are used. The layer size and the epoch number of each classifier, is obtained based on an optimization algorithm. Details about each of the three classifiers can be seen in Table 2 .

3. Voting between different networks: Three classifiers are trained based on the description in previous sections. Each network has a different performance between normal and abnormal signals. In order to better classification, we assigned a computed weight to each network. These weights are calculated based on the performance of each network in different training subsets.

Table 2. Characteristics of classifiers.

\begin{tabular}{lllll}
\hline $\begin{array}{l}\text { Neural } \\
\text { Network }\end{array}$ & $\begin{array}{l}\text { Layer } \\
\text { Size }\end{array}$ & $\begin{array}{l}\text { Train } \\
\text { Function }\end{array}$ & Epoch & $\begin{array}{l}\text { Type of signals } \\
\text { for training }\end{array}$ \\
\hline Net1 & {$[17,32]$} & $\begin{array}{l}\text { Sequential } \\
\text { order } \\
\text { weight/bias }\end{array}$ & 100 & $\begin{array}{l}\text { High \& Low } \\
\text { quality }\end{array}$ \\
Net2 & {$[19,11]$} & $\begin{array}{l}\text { Random } \\
\text { order } \\
\text { weight/bias } \\
\text { Random } \\
\text { order } \\
\text { weight/bias }\end{array}$ & 40 & High quality \\
Net3 & {$[19,11]$} & $\begin{array}{l}\text { High \& Low } \\
\text { quality }\end{array}$ \\
\hline
\end{tabular}




\section{Results}

In order to evaluate the performance of the presented method, the algorithm is submitted to PhysioNet/CinC Challenge 2016 web server as Close Source entries. Details about the characteristics of test set and the scoring can be found in [1].

Table 3 displays the performance of algorithm on the training set when it uses each of the classifiers separately, and the final result when using the voting systems for all three classifiers. Also, the optimal entry results in phase II of Challenge assessed on the hidden test set are shown.

Table 3. Results on training and hidden test sets

\begin{tabular}{|c|c|c|c|}
\hline classifier & Dataset & & validation \\
\hline Net1 & Training & $\mathrm{Se}=0.88$ & $\mathrm{Sp}=0.91$ Total $=0.90$ \\
\hline Net2 & Training & $\mathrm{Se}=0.94$ & .95 To \\
\hline Net3 & & $\mathrm{Se}=0.89$ & 92 Total $=0.91$ \\
\hline Voting & Tra & $\mathrm{Se}=0.96$ & $S p=0.98$ Total $=0.97$ \\
\hline Voting & $\begin{array}{l}\text { Hidden } \\
\text { test set }\end{array}$ & $\mathrm{Se}=0.81$ & 87 \\
\hline
\end{tabular}

\section{Discussion}

The main focus of this research is on modifying the algorithms used for segmentation, feature extraction and finally classification of heat sound recordings.

It is important to note that the results obtained using mentioned features depend strongly on segmentation. Since springer's algorithm does not segment correctly in some cycles of signal, a robust method is proposed to detect only cycles that are qualified enough for feature extraction. These mistakes are obvious where parts of s1 and s2 are mistaken as systole or diastole or vice versa (Figure 1). Moreover, the cycle selection method can recognize cycles that have noises mostly in systole and diastole components of a cycle which could not be filtered in the preprocessing stage and ignore them. A huge number of features are extracted. Perceptual features could better discriminate normal and abnormal recordings. But using all features as an input for a dimension reduction algorithm (FDA) a matrix is obtained to map all features into a new $2 \mathrm{D}$ space. The most common neural network type is multilayer-perceptron (MLP) associated with back propagation training algorithms. The use of this kind of neural networks did not result in a good classification in this project. The classifiers used are pattern recognition neural networks. This kind of classifier can adopt to the extracted features in a better way. But the performance of each is not good enough. Using voting system a better results both in the training and test sets are achieved. This makes our method suitable and efficient in the classification of heart sound recordings, as confirmed by the obtained results. It is suggested to employ better optimization algorithms in order to find the parameters of neural networks which will result in better classification performance.

\section{References}

[1] Liu C Y, Springer D B, Li Q, Moody B, Juan R A, Chorro F J, Castells F, Riog J M, Silva I, Johnson A E W, Syed Z, Schmidt S E, Panadaniil C D, Hadjileontiadis L, Naseri H, Moukadem A, Dieterlen A, Brandt C, Tang H, Samieinasab M, Samieinasab M R, Sameni R, Mark R G and Clifford G D. An open access database for the evaluation of heart sound algorithms. Physiol Meas 2016; 37 (in press).

[2] Naseri H, Homaeinezhad MR. Detection and boundary identification of phonocardiogram sounds using an expert frequency-energy based metric. Annals of biomedical engineering 2013;41(2):279-92.

[3] Springer DB, Tarassenko L, Clifford GD. Logistic regression-hsmm-based heart sound segmentation. IEEE Transactions on Biomedical Engineering 2016; 63(4):82232.

[4] Schmidt SE, Holst-Hansen C, Graff C, Toft E, Struijk JJ. Segmentation of heart sound recordings by a durationdependent hidden Markov model. Physiological Measurement 2010;31(4):513.

[5] Wang P, Kim Y, Soh CB. Feature extraction based on Melscaled wavelet transform for heart sound analysis. In2005 IEEE Engineering in Medicine and Biology 27th Annual Conference. 2006; 7572-7575.

[6] Naseri H, Homaeinezhad MR, Pourkhajeh H. Noise/spike detection in phonocardiogram signal as a cyclic random process with non-stationary period interval. Computers in biology and medicine 2013;43(9):1205-13.

[7] Ahlstrom C, Hult P, Rask P, Karlsson JE, Nylander E, Dahlström U, Ask P. Feature extraction for systolic heart murmur classification. Annals of biomedical engineering 2006;34(11):1666-77.

[8] Delgado-Trejos E, Quiceno-Manrique AF, Godino-Llorente JI, Blanco-Velasco M, Castellanos-Dominguez G. Digital auscultation analysis for heart murmur detection. Annals of biomedical engineering 2009;37(2):337-53.

Address for correspondence:

Mohammad Javad Mollakazemi molakazemi.javad@yahoo.com

K.N. Toosi University of Technology, No. 15, Pardis Street, MolaSadra Avenue, Vanak Sq.,Tehran, Iran. 